\title{
Inhalt - Contents, Vol. Supplement 1, 1997
}

\section{Sonderheft 1 zu Band 13, Januar 1997}

Gründungsherausgeber
A. Akovbiantz†, Zurich
H. Denck, Wien
K.-J. Paquet, Bad Kissingen
C. E. Zöckler, Bad Oeynhausen
Hauptschriftleiter
K.-J. Paquet, Bad Kissingen
Schriftleitung
Ch. E. Broelsch, Hamburg
M. W. Büchler, Bern
J. Horn, München
H. Säuberli, Baden
R. Schiessel, Wien
Assistenz:
K. Glaser, Innsbruck
Wissenschaftlicher Beirat
Deutschsprachiger Raum

H. M. Becker, München M. Betzler, Essen E. Bodner, Innsbruck H. Bosseckert, Jena H. Bülow, Schweinfurt R. Dölp, Fulda K. E. Frede, Basel

D. Fuchs, Innsbruck

A. Gangl, Wien

J. M. Hackl, Innsbruck

E. G. Hahn, Erlangen/Nümberg

J. Hauss, Leipzig

W.-D. Heine, Schweinfurt

P. Hermanek, Erlangen/Nürnberg

W. Hohenberger, Erlangen/Nürnberg

E. Klar, Heidelberg

W. Kozuschek, Bochum

F. Largiadèr, Zurich

S. Liebe, Rostock

H. Lippert, Magdeburg

P. Lukas, Innsbruck

P. Malfertheiner, Magdeburg

W. Mempel, München

J. Mössner, Leipzig

H. B. Reith, Würzburg

J. F. Riemann, Ludwigshafen 
H.-D. Saeger, Dresden

W. Schleinzer, Hamburg

W.-H. Schmiegel, Bochum

J. Schölmerich, Regensburg

P. Schweizer, Tubingen

M. Stolte, Bayreuth

M. Stahlschmidt, Mainz

M. Thelen, Mainz

A. Thiede, Würzburg

Bibliographischer Hinweis:

Die Zeitschrift ist in der Excepta-Medica-Datenbank EMBASE indexiert.

Ausland

J. Alexander-Williams, Birmingham

J. Gallinger, Moskau

J. M. Henderson, Cleveland

R. A. Hinder, Omaha

Y. Idezuki, Tokyo

I. Ihse, Lund

C. W. Imrie, Glasgow

G. G. Jamieson, Adelaide

S. L. Jensen, Århus

P. J. Kestens, Brüssel

B. Langer, Toronto

K. Maruyama, Tokyo

Ch. Meyer, Straßburg

W. C. Meyers, Durham

P. Morel, Genf

E. Moreno-Gonzáles, Madrid

V. Pegan, Ljubljana

A. Peracchia, Mailand

L. F. Rikkers, Omaha

J. Rodés, Barcelona

K. Sugimachi, Fukuoka

J. Terblanche, Kapstadt

A. L. Warshaw, Boston

Wu Zaide, Wuhan

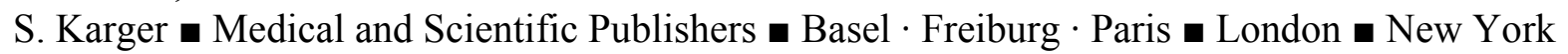

- New Delhi — Bangkok a Singapore a Tokyo a Sydney

Die Zeitschrift erscheint dreimonatlich; pro Jahr erscheint I Band zu je 4 Heften. Bezugspreis fur Jahrgang 12. 1996: DEM 195,-/SFr 153,- einschließlich MwSt., zuzüglich Post-gebühren. Der Abonnementpreis ist im voraus zahlbar. Das Abonnement der Zeitschrift läuft weiter, wenn es nicht spate-stens 4 Wochen vor Abschluß eines Bandes abbestellt wird.

Abonnementbestellungen können bei jeder Buchhandliing oder direkt beim Verlag aufgegeben werden: 
Bundesrepublik Deutschland: S. Karger GmbH, Lörracher Str. 16a, D-79115 Freiburg, Telefon (0761) 452070, Fax (0761) 45207 14, Postgiro München 614 40-808

Übrige Lander: S. Karger AC Allschwilerstr. 10, Postfach, CH-4009 Basel, Tel. (061)30611 11 , Fax (061)3061234 E-Mail Karger@Karger.ch

Anzeigen: S. Karger

Verlag für Medizin und Naturwissenschaften GmbH, Lörracher Str. 16a, D-79115 Freiburg, Tel. (07 61) 4520 70. Gültig ist die Preisliste Nr. 6 vom 1. Januar 1996.

Für den Inhalt außerhalb des redaktionellen Teiles (insbesonde-re Anzeigen.

Industrieinformationen, Pressezitate und Kon-greßinformationen) übernehmen Schriftleitung, Beirat und Verlag keine Gewähr.

Eine Markenbezeichnung kann warenzeichenrechtlich geschützt sein, auch wenn bei ihrer Verwendung in dieser Zeitschrift das Zeichen ${ }^{\circledR}$ oder ein anderer Hinweis auf etwa bestehende Schutzrechte fehlen sollte. Für Satzfehler, insbesondere bei Dosierungsangaben, wird keine Gewähr übernommen.

Die Zeitschrift sowie alle in ihr enthaltenen einzelnen Beiträge und Abbildungen sind urheberrechtlich geschützt. Jede Ver-wertung, die nicht ausdrücklich vom Urheberrechtsgesetz zu-gelassen ist, bedarf der vorherigen Zustimmung des Verlags. Das gilt insbesondere für Verviclfältigungen, Bearbeitungen, Übersetzungen, Mikroverfilmungen und die Einspeicherung und Verarbeitung in elektronischen Systemen. Fotokopien dürfen nur für den persönlichen Gebrauch als Einzelkopien hergestellt werden. Jede im Bereich eines gewerblichen Unternehmens zulässig hergestellte oder benutzte Kopie dient gewerblichen Zwecken gem. § 54(2) UrhG und verpflichtet zur Gebührenzahlung an die Verwertungsgesellschaft WORT, Abt. VG Wissenschaft, Goethestraße 49, D-80336 München.

Sonderheft 1 zu Band 13,

Januar 1997.

ISBN 3-8055-6461-9.

Ladenpreis des Sonderheftes:

DEM 55- inkl. MwSt.

Abonnenten erhalten es unberechnet

im Rahmen des Abonnements.

(C) Copyright 1997 by S. Karger

Verlag für Medizin und Naturwissenschaften $\mathrm{GmbH}$

Lörracher Straße 16a, D-79115 Freiburg

Verlagsleitung und presserechtlich verantwortlich: Sibylle Hopf

Redaktionsassistenz: Dr. Hannelore Tenckhoff Anzeigenverwaltung: Christiane Opitz

Produktionsleitung: Georg Brunner

Herstellung:

CW Niemeyer GmbH \& Co KG

Osterstraße 15-19, D-31785 Hameln

\section{KARGER}

(C) 1997 S. Karger GmbH, Freiburg Fax (07 61) 4520714 http://www.karger.ch

Inhalt $\square$ Contents

$\mathrm{C}^{1 / 8}$ rurgische $7 / 8>$

Band 13, Sonderheft 1, Januar 1997

Vol.13, Special Issue 1, January 1997 
Inhalt

Contents

VI Editorial

Hauss, J.; Mössner, J.; Witzigmann, H. (Leipzig)

1 Klassifikationen des exokrinen Pankreas-karzinoms

Wittekind, Ch. (Leipzig)

6 Diagnostik des Pankreaskarzinoms mittels Endoskopie und Endosonographie

Mössner, J. (Leipzig)

10 Nuklearmedizinische Diagnostik von Pankreas-tumoren mit der Positronen-Emissions-Tomographie (PET)

Knapp, W. H. (Leipzig)

121 st die chronische Pankreatitis eine Präkanzerose?

Keim, V.; Witzigmann, H. (Leipzig)

VI Editorial

Hauss, J.; Mössner, J.; Witzigmann, H. (Leipzig)

1 Classifications of Exocrine Pancreatic Carcinoma

Wittekind, Ch. (Leipzig)

6 Diagnostics of the Pancreatic Cancer by means of Endoscopy and Endosonography

Mössner, J. (Leipzig)

10 Nuclearmedical Diagnostics of the Pancreatic Tumors with the Positron Emission

Tomography (PET)

Knapp, W. H. (Leipzig)

12 Is the Chronic Pancreatitis a Precancerosis?

Keim, V.; Witzigmann, H. (Leipzig)

16 Molekularbiologie und Onkogenese des 16 Molecular Biology and Oncogenesis of

Pankreaskarzinoms Pancreatic Cancer

Friess, H.; Kleeff, J.; Berberat, P.; Büchler, MW. (Bern) ～Friess, H.; Kleeff, L; Berberat, P.;

Büchler, MW. (Bern)

24 Technik und Ergebnisse der chirurgischen Therapie beim Pankreaskarzinom

Kleeff, J.; Friess, H. Büchler, M. W. (Bern)

33 Stellenwert der Lymphknoten- und Binde-gewebsdissektion beim Pankreaskarzinom

Roder, J. D. (München)

24 Technique and Results of the Surgical Therapy of the Pancreatic Cancer

Kleeff, J.; Friess, H. Büchler, M. W. (Bern)

33 The Status of Lymph Node and Connective Tissue in Patients with Pancreatic Cancer

Roder, J. D. (München)

\section{KAIUiEK}

(C) 1997 S. Karger GmbH, Freiburg

Fax (0761)45207 14

http://www.karger.ch/journals/cga/cgacles.htm

36 Neuroendokrine Tumoren des Pankreas unter

besonderer Berücksichtigung der Insulinome

und Gastrinome 
Witzigmann, H.; Schwarz, R.; Kohlhaw, K.; Pohl, K.; Hauss, J. (Leipzig)

43 Palliative interventionelle Therapie beim Pankreaskarzinom mit Verschlußikterus

Möller, Th.; Lange, K. (Leipzig)

46 Adjuvante und neoadjuvante Therapieansätze in der Strahlentherapie des Pankreaskarzinoms Kamprad, F. (Leipzig)

50 Adjuvante Therapie des Pankreaskarzinoms

Gansauge, F.; Link, K. H.; Beger, H. G. (Ulm)

36 Neuroendocrine Pancreas Tumors with Particular Consideration of the Insulinoma and

Gastrinoma

Witzigmann, H.; Schwarz, R.; Kohlhaw, K.; Pohl, K.; Hauss, J. (Leipzig)

43 Palliative Interventional Therapy of Pancreatic Cancer Patients with Occlusive Jaundice Möller, Th.; Lange, K. (Leipzig)

46 Adjuvant and Neoadjuvant Possibilities in Radiation Therapy of Pancreatic Carcinoma

Kamprad, F. (Leipzig)

50 Adjuvant Therapy in Pancreatic Cancer

Gansauge, F.; Link, K. H.; Beger, H. G. (Ulm)

II Impressum

II Imprint

\section{KARGEÍl}

(C) 1997 S. Karger GmbH, Freiburg

Fax (0761) 4520714

http://www.kager.ch/journals/cga/cgades.htm

Die Natur ergreift immer die Partei des versteckten Fehlers.

Gesetz.)

irkung - auch auf Enterokokken- nicht sein darf. Vor allem in der Klinik nicht.

Murphys Gesetz beschreibt die Gesetzmäßigkeit des Zufalls: Wenn etwas schiefgehen kann, dann wird es auch schicfgehen! (If anything can go wrong, it will.)

Die Infektíonsprophylaxe mit Baypen setzt dieses Gesetz und seine Gesetzmäßigkeiten

weitestgehend außer Kraft. Auf-

und seiner breiten Einsatzmöglichkeit, ohne Gefahr der Induktion von Parallelresistenzen, erhöht Baypen die Sicher-heits-Standards bei Bauchraum-Operationen und Eingriffen im

gynäkologischen Bereich.

Denn - auch dies ist ein Gesetz: Es kann nicht sein, was

Bayperf $2 g$

tspektrum-Penicillin

5 fusion; auch i v Injektion und i.m, Injektion. I g1/8B «rdl Kurzinfusiort ca. 10-15 Minut $\beta$ n.

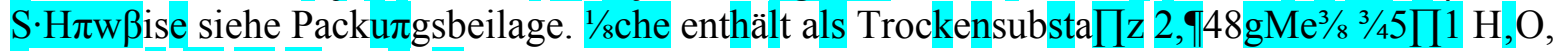
entsprechend $2 \mathrm{~g}$ Mezloallm,

* SfLeverkuse $\pi \quad$ zuyii

e»* $\cdot \mathrm{i}$

. um «

1/8rphy’s Gesetz: deutschsprachige Rechte Goldmann Verlag, Müncher 
Baypen" Wirkstoff: Mezlocillin. Breitspektrum-Antibiotikum. Zusammensetzung: Arzmeilich wirksamer Bestandteil: Baypen 0,5/1/2/3/4/5 g: 1 Fl. Trockensubst. enth. 0,537/1,074/2,148/ 3,222/4,296/5,37 g Mezlocillin-Natrium 17/80 e $\pi$ tspr. 0,5/1/2/3/4/5 g Mezlocillin. Weitere Bestandteile: Baypen 0,5/1/2/3/4/5 g: $1 \mathrm{Fl}$. Lösungsmitteïenth. Wasserfür Injektionszwecke. Anwendungsgebiete: Durch Mezlocillin-empfi $\pi$ dliche Erreger verursachte Infektionen - auch Mischinfektíonen - verschiedenster Lokalisation und Intensität; Infektionen Oder drohende Infektionsgefahr bei Patiente $\pi$ mit reduzierter Abwehrlage; perioperative Kurzzeitprophylaxe bzw. Frühtherapie in Abhängigkeit von Risikofaktoren und Kontaminationsgefãhrdu $g$. Bei drohender bakterieller Allgemeininfektion und/oder unbekannten Oder weniger empfindlichen Erregern sowie bei Super- oder Mischinfektionen ggf. Kombitationstherapie mit ande-ren bakterizid wirksamen Substanzen. Gegenanzeigen: Penicillinüberempfindlichkeit; bei Überempfindlichkeit gegen a $\pi$ dere $\beta$-Lactam-Antibiotika, z.B. Cephalosporine, mögliche

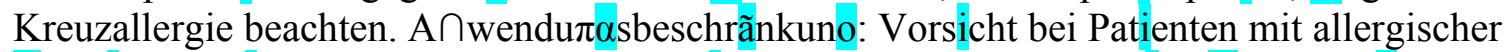
Reaktionsbereitschaft, z.B. Asthma bronchiale, Heuschnupfen, Urtikaria, wegen erhõhter Disposition zu Überempfindlichkeitsreaktionen. Baypen ist plazentagängig und tritt in die Muttermilch über: Anwendung wãhrend der gesamten Schwangerschaft bei entsprechender Indication môglich. Nebenwirkungen: Magen-Darm-Trakt: Gelegentlich Übelkeit, Erbrechen, Meteorismus, weiche Stühle oder Durchfâlle bis hin zu einer antibiotikabedingten lebensbedrohlichen pseumembra ösen Enterokolitis. Überempfindlichkeitsreaktionen: Gelegentlich Hautreaktionen (z. B. Exanthem, Juckreiz). Bei Urtikaria Baypen absetzen uीd Behandlung nicht mit anderen Penicillinen fortsetzen. Selten Eosinophilie, Arzneimittelfieber, akute interstitielle Nephritis, Vaskulitis. In Einzelfällen Lyell-, Stevens-Johnson-Syndrom, anaphylaktischer Schock. Blut: Bei Hochdosierung selten Thrombozytenfunktionsstörungen mit Verlängerung der Blutungszeit, Purpura oder Schleimhautblutungen, vor allem bei Patienten mit stark eingeschränkter Nierenfunktion. Bei klinisch manifesten Blutunge $\cap$ Baypen absetzen. In Einzelfällen Leukozytendepressio $\cap$ bis hin zur Agranulozytose, Ariämie oder Thrombozytopenie. Bei lãngerer Behandlung Kontrolle des Blutbildes. ZNS: Anwendung sehr hoher i.v. Dosen von Penicillinen: zentralnervöse Erregungszustände, Myoklonien und Krämpfe. Nicht angepaßte Dosierung bei eingeschränkter Nierenfunktion sowie erhõhte Liquorgängigkeit infolge Erkrankungen des Gehirns fõrdern die zerebrale Krampfneigung. Geschmacksirritationen können bei Einhalten einer Ihjektionsgeschwindigkeit von ca. $5 \mathrm{ml} / \mathrm{min}$ verhindert werden. Lokale Reaktionen: Selten Thrombophlebitiden oder Schmerzen am Injektionsort. Laborwerte: Vorübergehender Transaminasenanstieg (SGOT, SGPT) oder Erhõhung der alkalischen Phosphatase, Hypokaliämie, Anstieg von Bilirubin, Kreatinin und Reststickstoff, positiver Coombs-Test. Hinweis: Bei langfristiger oder wiederholter Anwendung Superinfektionen mit resistenten Bakterien oder mit Sproßpilzen. Wechselwirkungen mit anderen Mittein: Hochdosiert Heparin, orale Antikoagulanzien oder Thrombozytenaggregationshemmer: Gerinnungsparameter regelmãßig überwachen. Probenecid: Hemmung der renalen Ausscheidung und höhere Serumkonzentrationen von Mezlocillin. Andere Antibiotika: Aminoglykoside und penicillinasefste Penicilline können ei $\pi$ en synergistischen Effekt haben. Nichtdepolarisierende Muskelrelaxanzien: Vertiefung und Verlängerung der neuromuskulãren Blockade bei Anwendung von Mezlocillin unter oder unmittelbar nach Operationen. Methotrexat: Überwactiung der ev. erhõhten Methotrexatspiegel. Ninhydrinprobe, nichtenzymatísche Harnzuckerreaktion, Urobilinogennachweis sowie die Proteinbestimmung im Harn können falsch positiv ausfallen. 
Dosierung: Therapie: Erwachsene und Jugendliche ab 14 Jahren erhalten als Normdosis täglich 80-150 mg/kg KG, max. 200 - 300 mg/kg KG; Kinder und Säuglinge: 3 x 75 mg/kg KG; Neuund Frühgeborene: 2 x $75 \mathrm{mg} / \mathrm{kg} \mathrm{KG}$. Bei

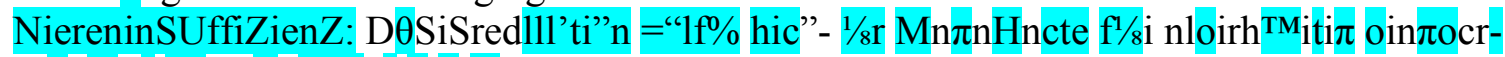
$\operatorname{hrdn} 1 / 8 \mathrm{r}$ I ohprf, inL.1/8n- u/oita-

postoperativ. Art der Anwendung: Herstellung: Trockensubst. ca. 10\%ig in Wasser für Injektionszwecke lösen und

alsbald verwenden. Intrave $\pi$ õse Injektion ( $5 \mathrm{ml} / \mathrm{min}$ ); intravenöse Infusion: Kurzinf. ca. 20 30min oder nach

Verdünnung mit geeigneten Infusionslõsungen, Dauertropfinfusion. Intramuskulãre Injektion: Iniektionsvolumina

beachten. Dauer der Anwendung: Die Behandlungsdauer richtet sich nach der

Schwereder Infektion, mind. 3 Tage über Entfieberung hinaus; Streptokokkeninfekt. i-5/8 $\wedge ¥ \sim 1$

mind. 10 Tage; perioperative Kurzzeitprophylaxe: max. 3 Tage. Handelsforr $\cap e n: \Gamma$

Baypen 0,5/1/2/3/4/5 g: 5 Fl. Trockensubst. und Lösungsmittel (N2). Anstaltspacku $\pi g e n$.

Verschreibungspflichtig. Stand: D/6; Januar 1997. Bayer Vital GmbH \& Co. KG,

51368 Leverkusen

Chir Gastroenterol 1997;13(suppl 1):VI

EDITORIAL

J. Hauss, J. Mössner, H, Witzigmann, Leipzig

Das Pankreaskarzinom hat mit einer mitt-leren Uberlebenszeit von 5 Monaten eine der schlechtesten Prognosen aller malig-nen Neoplasien des Menschen. Die Inzi-denz ist in den westlichen industrialisier-ten Ländern signifikant angestiegen, offensichtlich infolge einer Verlängerung der mittleren Lebenserwartung und einer Verbesserung der diagnostischen Möglichkeiten. Derzeit sterben etwa 10 von 100000 Menschen an den Folgen eines

Pankreaskarzinoms. Es ist somit die vierthäufigste Todesursache durch malig-ne Neubildungen. Etwa 20\% der Pankreaskarzinome sind zum Zeitpunkt der Diagnose resektabel. Die 5-JahresÜberlebensrate bei Patienten mit resektablen Tumoren liegt bei $15-20 \%$ und bei alien Pankreaskarzinom-patienten unter 1\%. Gründe für die schlechte Prognose sind die Lage im Retroperitoneum mit eingeschränkten Re-sektionsgrenzen, die spate Symptomatik und Diagnose und das aggressive Tumor-wachstum. Neuere Daten deuten darauf hin, daß molekulare Störungen in den Pankreaskarzinomzellen eine wesentliche Ursache für ihre klinische Aggressivität darstellen.

Eine Verbesserung der schlechten Prognose ist in erster Linie von einer Diagnose im Frühstadium zu erwarten. Deshalb liegt die Herausforderung an die präopera-tive Diagnostik in der Früherkennung kleinerer Tumoren und in der Differenzie-rung zwischen Malignom und lokaler Ent-zündung.

Die operative Entfernung des Pankreaskarzinoms ist immer noch der einzige ku-rative Therapieansatz. Die Wertigkeit einer erweiterten Lymphknoten- und Binde-gewebsdissektion $\mathrm{mu}$ noch in prospekti-ven Studien geprüft werden. Die adjuvan-te Radiochemotherapie führt offenbar zu einer signifikanten Verlängerung der me-dianen Uberlebenszeit. Von präoperativen Therapieverfahren, welche derzeit in Studien geprüft werden, ist ebenfalls eine ef-fektive Prognoseverbesserung zu erwarten. Die Intention dieses Supplements ist es, den heutigen Stand der interdiszi-plinären Diagnostik und Therapie des Pankreaskarzinoms unter besonderer 
Berücksichtigung der Perspektiven zu präsentieren. Die Beiträge basieren auf Vorträgen, die anläßlich eines Symposiums am 6. September 1996 an der Univer-sität Leipzig gehalten wurden.

\section{KARGEH}

(C) 1997 S. Karger GmbH. Freiburg Fax (0761) 4520714 http://www.karger.ch

Dr. H. Witzigmann Chirurgische Klinik und Poliklinik I Zentrum Chirurgie Universitätsklinikum Leipzig Liebigstraße 27 D-04103 Leipzig 